ORIGINAL ARTICLE

\title{
A model for determining the optimum histology of sentinel lymph nodes in breast cancer
}

\section{G Cserni}

Correspondence to: Dr G Cserni, Department of Pathology, Bács-Kiskun County Teaching Hospital, Nyiri ut 38, POB 149 , Kecskemét, $\mathrm{H}-6000$, Hungary;

cserni@freemail.hu

Accepted for publication 22 December 2003

\begin{abstract}
Aims: To create and use a geometrical model for sentinel lymph node (SLN) histopathology in breast cancer.

Methods: The model involves a spherical metastasis randomly situated in an SLN. Two extreme situations are taken as the starting points. In one of these, the metastasis is seen in its largest dimension, whereas in the other it is only just visible, approximating $0 \mathrm{~mm}$ in size. Intermediate positions are analysed, with different metastasis sizes and different distances between the levels assessed by histology.

Results: The findings suggest that sections taken $1 \mathrm{~mm}$ apart afford a reasonable means of identifying almost all metastases measuring $>2 \mathrm{~mm}$ (referred to as macrometastases here). For nearly all micrometastases to be identified correctly according to the current TNM definitions (that is, metastases $>0.2 \mathrm{~mm}$ ), a step sectioning protocol with levels of $250 \mu \mathrm{m}$ or $200 \mu \mathrm{m}$ would be adequate.

Conclusions: SLNs are the most likely sites of nodal metastasis. Macrometastases are of recognised prognostic relevance so that all should be identified, preferably correctly as macrometastases; an assessment of levels $1 \mathrm{~mm}$ apart appears satisfactory and sufficient for this aim. SLNs also offer an ideal method for the study of the significance of micrometastases; for this, step sections separated by 200 or $250 \mu \mathrm{m}$ are a good choice.
\end{abstract}

S entinel lymph nodes (SLNs) are the most likely sites of lymphogenic metastasis in breast cancer, as suggested in numerous reports since the publication of the milestone paper by Giuliano et al. ${ }^{1}$ This has been substantiated by studies involving a similarly detailed histopathological investigation of both SLNs and non-SLNs. ${ }^{2-5}$ SLN biopsy may lead to the upstaging of patients with breast cancer in a considerable proportion of cases, depending on the depth of investigation. ${ }^{6}$ Most of the metastases revealed by a more detailed investigation are micrometastases (with an upper inclusive size of $2 \mathrm{~mm}$ ) or isolated tumour cells (ITCs, with an upper inclusive size of $0.2 \mathrm{~mm}$ ), as defined by the TNM classification, ${ }^{7}$ but some may be larger. ${ }^{18}$

Because most of the prognostic information relating to nodal status stemmed from the assessments of standard single haematoxylin and eosin stained sections of the lymph nodes, it is obvious that most of the metastases identified were larger than micrometastases. (These will be referred to here as macrometastases.) Such metastases are associated with the worse prognosis attributed to the node positive status.

Micrometastases are defined in various ways in the literature, ${ }^{9}$ but the most common definition is that introduced by Huvos and colleagues ${ }^{10}$-an upper limit of $2 \mathrm{~mm}$. This is the size adopted in the TNM classification of breast tumours, which has also recently been associated with a lower non-inclusive size limit of $0.2 \mathrm{~mm} .{ }^{71}$ Anything below this size (and with no metastatic activity) is referred to as an ITC, and is not regarded as nodal involvement for staging and treatment purposes. ${ }^{711}{ }^{12}$ In most publications before 2002, the range of micrometastases includes the current ITC category. Their prognostic relevance is uncertain and debated. In his often quoted review, Dowlatshahi suggested that only larger studies with a substantial follow up have demonstrated a worse prognosis for patients with micrometastases. ${ }^{9}$ However, a recent study, in which there was such a follow up and which was subjected to multivariate analysis, revealed that the prognosis of patients with occult metastases (many of which should have been called micrometastases at the time of Dowlatshahi's review ${ }^{9}$ ) disclosed by immunohistochemistry (IHC) was no worse than that for patients without nodal involvement. ${ }^{13}$

"If one considers the consequences of nodal staging, it is not only the identification of the nodal cancer cells that is important, but also the classification of this nodal involvement into the categories of isolated tumour cells, micrometastasis, and macrometastasis"

Methods for the pathological investigation of SLNs vary considerably; in general, the more sectioning levels investigated, the more ITCs or metastases can be identified. ${ }^{6}{ }^{14}$ If one considers the consequences of nodal staging, it is not only the identification of the nodal cancer cells that is important, but also the classification of this nodal involvement into the categories of ITC (pNO), micrometastasis (pNlmi), and macrometastasis (pNl). For example, a metastasis diagnosed as a micrometastasis in one section may be graded as macrometastasis at a deeper level, but only if this deeper level is also investigated. The differentiation between micrometastases and macrometastases may not seem important from the point of view of staging (both are N1), but owing to the debated prognostic relevance of micrometastases, and suggestions in the literature that they may not be associated with further echelon lymph node metastasis, this distinction might be important for decisions about locoregional treatment.

The European working group for breast screening pathology has reviewed current data to allow the formulation of the European guidelines for SLN specimens, ${ }^{6}$ and has accepted that a minimum standard of SLN assessment should aim at the identification of all macrometastases, ${ }^{15}$ an aim that is not

Abbreviations: IHC, immunohistochemistry; ITC, isolated tumour cell; SLN, sentinel lymph node 


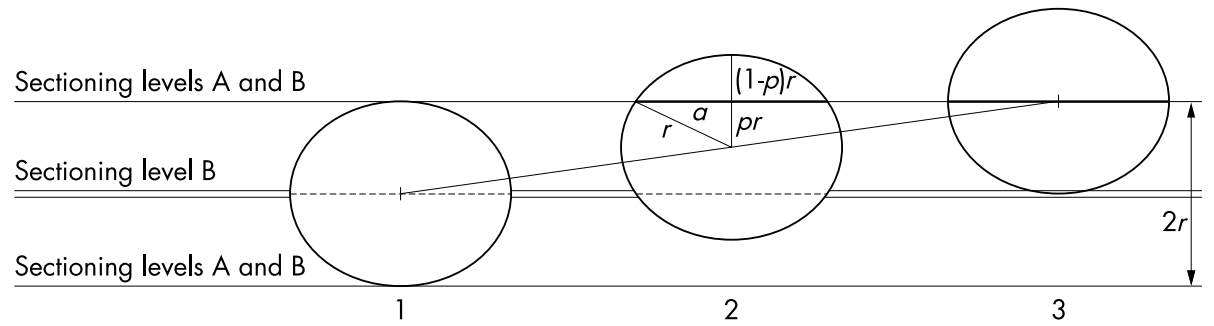

Figure 1 The model of sentinel lymph node step sectioning and detection of a metastasis of a given size. Metastases are represented by circles. Single horizontal lines relate to sectioning protocol A (sectioning levels equalling the cutoff size of a given category-for example, $2 \mathrm{~mm}$ for micrometastases - and also the diameter of the metastasis in this specific example), whereas the double line relates to the additional sectioning level in protocol B (sectioning levels equalling half the category defining the cutoff size, and also the radius of the metastasis in this example), which also includes the planes of protocol $A$. The numbers at the bottom denote specific situations: numbers 1 and 3 are two extreme positions, whereas number 2 reflects the position midway between the extremes. The segment joining the centres of the metastases in situations 1 and 3 shows the possible positions of the centre of a metastasis between the extremes. The thick segments in situations 2 and 3 represent the observed dimension of the metastasis as revealed by both protocol $A$ and $B$, whereas the broken segments in situations 1 and 2 refer to the dimension of the metastasis as disclosed by the additional levels of protocol $B$. In situation 2, $r$ is the radius of the metastasis, pr is the proportion of the radius separating the plane of the section from the centre of the metastasis, $(1-p) r$ is the distance between the plane of the section and the edge of the metastasis, and $a$ is half the length of the thick segment (the identified size of the metastasis). Note that $a$ is also the length of one of the perpendicular sides of the rectangular triangle with further sides $p r$ and $r$, and that $p r$ is equal to $r / 2$ in situation 2 (for further details, see the text).

achieved with all current protocols. ${ }^{16}$ It has also been suggested that it would be optimum for micrometastases to be identified also.

This article describes a geometrical model for the identification and adequate characterisation of SLN involvement, on the basis of which a better method can be recommended for the SLN work up.

\section{METHODS AND RESULTS}

A model relating to the probability of identifying a lymph node metastasis of given size by different step and/or serial sectioning methods was reported earlier. ${ }^{17}$ This model assumed that metastases were spherical and randomly distributed in the SLN, and for convenience only a single metastasis was hypothesised, because this is often the case. The same assumptions are used in the current model.

A metastasis measuring $2 \mathrm{~mm}$ in diameter will first be considered because this is the upper limit of the micrometastasis category, and anything larger would be graded as a macrometastasis. Theoretically, a spherical metastasis of this maximum size could be identified by serially sectioning the SLN at a distance of $2 \mathrm{~mm}$ (sectioning level A), if we consider that the thickness of a section is $5 \mu \mathrm{m}$. However, in one extreme situation this metastasis would be identified as a $2 \mathrm{~mm}$ metastasis, whereas in the other extreme situation it would be almost completely missed, and identified at best as an ITC (fig 1, situations 3 and 1, respectively.) Between these extremes, the micrometastasis could be identified as having any size between just over $0 \mathrm{~mm}$ and $2 \mathrm{~mm}$.

The centre of the metastasis shown in fig 1 can be anywhere on the segment joining the centres of the circles in situations 1 and 3. If we consider the triangle with sides $a, p r$, and $r$, the $2 a$ long thick segment represents the size of the metastasis as measured in sectioning plane A. This length can be determined on the basis of Pythagoras's theorem as:

$$
2 a=2\left[r^{2}-(p r)^{2}\right]^{1 / 2}(1)
$$

and can be plotted against the length of segment $p r$ as shown in fig 2.

With sections at a distance of $1 \mathrm{~mm}(r$, protocol B in fig 1$)$, the two extreme positions of the metastasis will be shown by diagrams 1 and 2 in fig 1 . The metastasis will be identified with its maximum dimension in the first case (the broken segment in position 1) and with smaller dimensions in the second (the thick and broken segments in position 2). If the centre of the metastasis is moved in either direction from position 2 along the segment joining the centres of circles 1 and 3, one or the other of the thick or broken segments in the sectioning level B planes will increase. In this case, the size of the metastasis will be identified as anything between $2 \mathrm{~mm}$ and $1.73 \mathrm{~mm}$, as shown in fig 2 .

Because the precision of tumour and metastasis dimension measurements in microscopy is often to the nearest $100 \mu \mathrm{m}$, a macrometastasis would have a maximum dimension of $2.1 \mathrm{~mm}$ or above. For the same model of sectioning at $2 \mathrm{~mm}$ or at $1 \mathrm{~mm}$, the extreme positions of this metastasis are shown in fig 3 and the measurable dimensions of the metastasis are illustrated in fig 4 . The lowest apparent sizes of a metastasis with a diameter of $2.1 \mathrm{~mm}$ with different stepwise sectioning procedures can also be read off the graph. The segment $p r$ in position 2 in figs 1 and 3 is always half way between the levels assessed-for example, a step sectioning protocol with levels separated by $500 \mu \mathrm{m}$ will identify a metastasis of $2.1 \mathrm{~mm}$ in diameter as a metastasis between $2.1 \mathrm{~mm}$ and $2.04 \mathrm{~mm}$ (with this last figure corresponding to the value of the graph at $\mathrm{x}=0.25$ and to the lowest apparent size; this rounds down to $2 \mathrm{~mm}$ ), whereas a step sectioning protocol with levels separated by $250 \mu \mathrm{m}$ would identify the same metastasis as a $2.1 \mathrm{~mm}$ metastasis (if we round up all values to the nearest $100 \mu \mathrm{m}$ ). Therefore, the smallest macrometastasis would always be identified as a macrometastasis with this last step sectioning procedure. For these calculations, $p r$ in equation 1 can be replaced by $d / 2$,

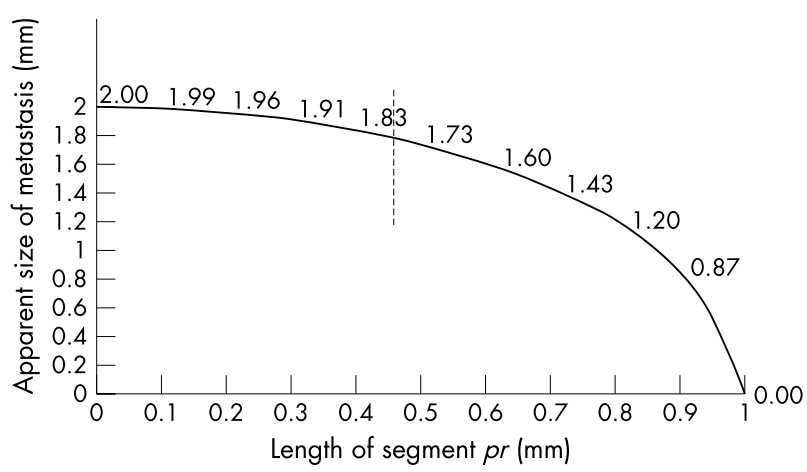

Figure 2 Observed sizes of a $2 \mathrm{~mm}$ diameter metastasis as measured in the plane of section $A$ as a function of the length of segment pr from fig 1, when the distance between the sections is $2 \mathrm{~mm}$. Situations 1 and 3 in fig 1 correspond to the points at the right and left edges of the graph, respectively, whereas situation 2 at the middle is highlighted by the broken segment. 
Sectioning level B

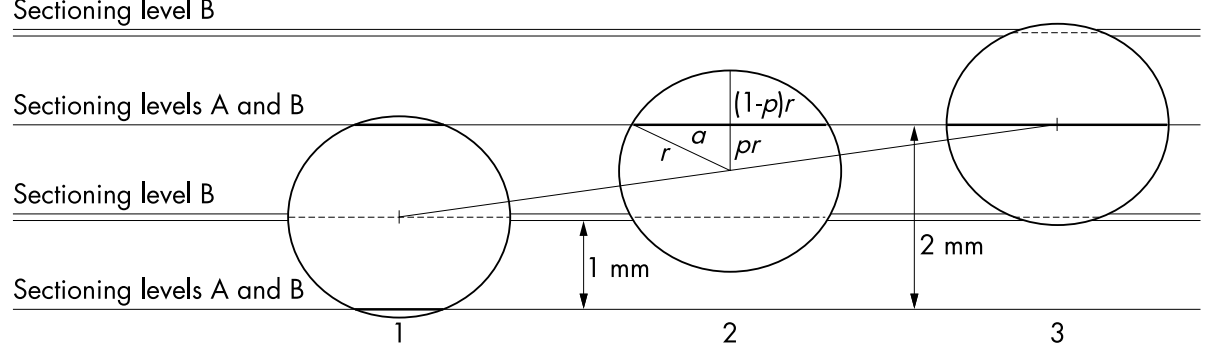

Figure 3 Model of a macrometastasis just over $2 \mathrm{~mm}$ in diameter $(2.1 \mathrm{~mm}$ in the example) in distinct, special situations. Displacement of the circle representing the metastasis along the segment joining the centres of circles 1 and 3 (positions with minimum and maximum measured sizes on sectioning planes $A$, respectively) from position 2 towards either position 1 or position 3 results in a larger measured size of the metastasis at sectioning level B. If segment $p r$ is half the stepwise distance in sectioning protocol B, and consequently a quarter of the category cutoff value $(2 \mathrm{~mm}$ for micrometastases), segment $2 a$ (thick segment in position 2) would be the lowest apparent size of the given metastasis with sectioning protocol B. For details, see fig 1 and the text. The distance between the sectioning levels is $2 \mathrm{~mm}$ in protocol A and $1 \mathrm{~mm}$ in protocol B.

where $d$ is the distance between sectioning levels. This would result in the equation below:

$$
2 a=2\left[r^{2}-(d / 2)^{2}\right]^{1 / 2}(2) .
$$

Table 1 illustrates the possibilities of misinterpreting a macrometastasis as a micrometastasis as reflected by its size and the different step sectioning protocols. Protocols with sectioning levels separated by more than $2 \mathrm{~mm}$ are also listed, because lymph nodes are sometimes sliced with this degree of precision, and slices of $3 \mathrm{~mm}$ are not rare in practice. As shown in table 1 , slices larger than $2 \mathrm{~mm}$ may miss some of the macrometastases, as indicated in connection with the probability model of Meyer. ${ }^{17}$

For micrometastases, the reasoning is very similar. According to the current TNM classification, the size of micrometastases ranges from $>0.2 \mathrm{~mm}$ up to $2 \mathrm{~mm}^{711}$ Accordingly, if one wishes to detect these metastases as micrometastases and not as ITCs, on analogy with the earlier considerations, the lower cutoff value of $0.2 \mathrm{~mm}$ may be applied for this category. Table 2 shows the possibilities of misinterpreting a micrometastasis as something smaller than this category. The possible misinterpretation of macrometastases as micrometastases has been referred to in connection with table 1 .

\section{DISCUSSION}

Because SLNs are the most likely sites of lymphogenic metastasis and the nodal status is an important prognostic

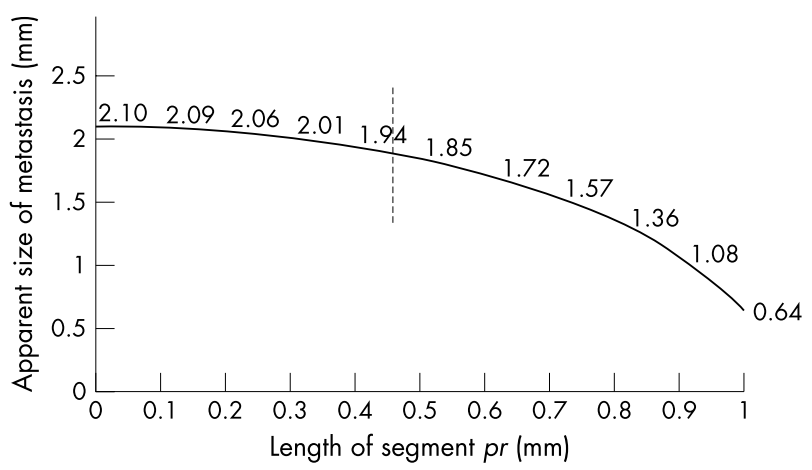

Figure 4 Observed sizes of a $2.1 \mathrm{~mm}$ diameter metastasis as measured in the plane of section $A$ as a function of the length of segment pr from fig 3, when the distance between the sections is $2 \mathrm{~mm}$. Situation 1 in fig 3 is shown by the point at the right edge of the graph $(0.64 \mathrm{~mm}$ lowest apparent size), situation 3 with the maximum size identified is represented at the left edge, and situation 2 at the middle is highlighted by the broken line. With a sectioning distance of $1 \mathrm{~mm}$, the apparent size can vary between $2.1 \mathrm{~mm}$ and $1.85 \mathrm{~mm}$ (broken line). factor in breast cancer, the investigation of SLNs must be more detailed than that of unselected lymph nodes from the axilla. The prognostic relevance of micrometastases is still disputed in the literature, and thus the identification of metastases larger than this $(>2 \mathrm{~mm})$ should be the minimum standard in SLN histopathology. This target is not attained by many of the currently used histology protocols. However, it is not enough simply to identify a macrometastasis as nodal involvement; in the optimum situation, we should be able to categorise it as a macrometastasis.

With the model described here, all macrometastases can be correctly identified as such if the step sectioning protocol allows the examination of levels separated by $250 \mu \mathrm{m}$ (table 2) (this is like the protocol we apply at our institution ${ }^{8}$ ). On the other hand, a protocol that has a workload $25 \%$ of this (the examination of levels separated by $1 \mathrm{~mm}$ ) permits the recognition of all macrometastases measuring $>2.2 \mathrm{~mm}$, and its error rate therefore seems acceptably low. Levels separated by $1 \mathrm{~mm}$ can be easily assessed by a combination of slicing SLNs into parallel pieces 2-3 mm in thickness, and then step sectioning them. Because only a minority of macrometastases fall into the range from $>2 \mathrm{~mm}$ up to $2.2 \mathrm{~mm}$, and even some of these can be picked up as macrometastases by chance, this protocol can be highly recommended on the basis of the model used.

\section{"Sentinel lymph node biopsy seems an ideal tool for the study of prognosis related to micrometastases"}

Although nodal micrometastases are not fully recognised to have relevant prognostic value, they are classified as $\mathrm{pNl}$ in the TNM system, and hence an optimum staging should aim to identify them. SLN biopsy seems an ideal tool for the study of prognosis related to micrometastases. It is evident that this would involve a much greater workload, and the protocol recommended above for macrometastases (1 mm between levels) can adequately classify only those micrometastases measuring $>1.1 \mathrm{~mm}$ (equation 2; value not shown in table 2) on the basis of the model; smaller metastases would be identified as micrometastases only by chance. This is why a smaller distance between the levels assessed should be considered for step sectioning. Table 2 suggests that a distance of $200 \mu \mathrm{m}$ between levels would not lead to the classification of micrometastases as ITCs, and even a $250 \mu \mathrm{m}$ step sectioning protocol would have only a small rate of error and misclassify some of the $0.3 \mathrm{~mm}$ metastases as ITCs.

Previous studies in which prognostic relevance was associated with micrometastases did not generally use a 
Table 1 Lowest apparent sizes of spherical metastases of given diameters with step sectioning at given distances

\begin{tabular}{|c|c|c|c|c|c|c|c|c|c|c|c|}
\hline \multirow[b]{2}{*}{ Distance between sections } & \multicolumn{11}{|c|}{ Metastasis size (diameter in $\mathrm{mm}$ ) } \\
\hline & 2.0 & 2.1 & 2.2 & 2.3 & 2.4 & 2.5 & 2.6 & 2.7 & 2.8 & 2.9 & 3.0 \\
\hline $3 \mathrm{~mm}$ & 0 & 0 & 0 & 0 & 0 & 0 & 0 & 0 & 0 & 0 & 0 \\
\hline $2.5 \mathrm{~mm}$ & 0 & 0 & 0 & 0 & 0 & 0 & 0.7 & 1.0 & 1.3 & 1.5 & 1.7 \\
\hline $2 \mathrm{~mm}$ & 0 (ITC) & 0.6 & 0.9 & 1.1 & 1.3 & 1.5 & 1.7 & 1.8 & 2.0 & 2.1 & 2.2 \\
\hline $1 \mathrm{~mm}$ & 1.7 & 1.8 & 2.0 & 2.1 & 2.2 & 2.3 & 2.4 & 2.5 & 2.6 & 2.7 & 2.8 \\
\hline $0.5 \mathrm{~mm}$ & 1.9 & 2.0 & 2.1 & 2.2 & 2.3 & 2.4 & 2.6 & 2.7 & 2.8 & 2.9 & 3.0 \\
\hline $0.25 \mathrm{~mm}$ & 2.0 & 2.1 & 2.2 & 2.3 & 2.4 & 2.5 & 2.6 & 2.7 & 2.8 & 2.9 & 3.0 \\
\hline $0.2 \mathrm{~mm}$ & 2.0 & 2.1 & 2.2 & 2.3 & 2.4 & 2.5 & 2.6 & 2.7 & 2.8 & 2.9 & 3.0 \\
\hline $0.15 \mathrm{~mm}$ & 2.0 & 2.1 & 2.2 & 2.3 & 2.4 & 2.5 & 2.6 & 2.7 & 2.8 & 2.9 & 3.0 \\
\hline \multicolumn{12}{|c|}{$\begin{array}{l}\text { The cells that are below the stepped line correctly identify the macrometastases in the column headings as such, } \\
\text { whereas the cells above this line can identify them as micrometastases or miss them altogether, although by chance } \\
\text { they can also find them and categorise them correctly. The value } 0 \text { (ITC) suggests that a metastasis with a diameter } \\
\text { equal to the distance between the levels could be seen at least as an ITC, because the thickness of the sections is not } \\
\text { accounted for in this model; however, this value rounds to } 0 \text { in the table. (For details, see Methods and Results } \\
\text { sections.) } \\
\text { ITC, isolated tumour cells. }\end{array}$} \\
\hline
\end{tabular}

detailed histology protocol in which levels separated by $1 \mathrm{~mm}$ or less were investigated. Accordingly, some of their micrometastases could have been larger, but not classified as such.

The smallest degree of SLN involvement is that by ITCs. These are currently not considered metastases, and it is recommended that they be ignored for treatment purposes. ${ }^{611}{ }^{12}$ Their identification is not and cannot be the aim of a pathological protocol, ${ }^{18}{ }^{19}$ but more detailed protocols will naturally identify them more frequently, ${ }^{8}$ and they should be adequately reported, as stressed by the European working group for breast screening pathology. ${ }^{15}$

The conclusions formulated above have been reached on the basis of a simple model, which can be criticised on several levels. Although larger metastases are not infrequently globoid, they are almost never exactly spherical, and all pathologists have encountered irregularly shaped metastases-for example, single cell pattern metastases of lobular carcinomas. This criticism of the shape may especially hold true for micrometastases, which are often seen along the subcapsular sinuses. Nevertheless, it is felt that this is a good approximation for macrometastases, and the suggested protocol is a reasonable way of correctly identifying nearly all metastases measuring $>2 \mathrm{~mm}$ as macrometastases.

We were perhaps the first to report that metastases are not randomly distributed in SLNs, ${ }^{20}$ and this observation has recently been reinforced. ${ }^{21}$ Metastases are preferentially found around the inflow junction of the tumour draining lymphatic channel; this can be discerned either intraoperatively ${ }^{21}$ by identifying the blue lymphatic itself, together with the lymph node, or by picking up the area of the SLN with the highest $\gamma$ counts by the probe. Alternatively, removal of the SLN with the lymphatic itself allows identification of the inflow point in the pathology department. ${ }^{22}$ This knowledge may promote better use of the available resources.

The recommended protocols may seem very extensive, but can be reduced. Macroscopically negative SLNs may be halved or sliced for intraoperative assessment. We favour imprint cytology, ${ }^{23} 24$ but frozen sectioning can be equally accurate. ${ }^{6}$ Targeting the inflow point of the tumour draining lymphatic vessel can make this assessment more reliable. ${ }^{22}$ If the lymph node is positive, confirmation by a section might be sufficient. The availability of a few sections at steps of $1 \mathrm{~mm}$ for macrometastases, or at 200 or $250 \mu \mathrm{m}$ for micrometastases (depending on the aim of the studies, or on the resources), may obviate the need for further sections in the event of positive findings. Such a stepwise approach may reduce the costs of a routinely applied intensive work up. However, with a stepwise approach or less intensive approaches, the identification of a small metastasis should be confirmed at deeper levels to rule out a possibly larger size.

The reported role of IHC is also controversial. We find it very useful, as do many others. ${ }^{142526}$ It may help with the identification not only of ITC, but also of micrometastases, and in a stepwise approach it may point to the tip of a larger

Table 2 Lowest apparent sizes of spherical metastases of given diameters with step sectioning at given distances

\begin{tabular}{|c|c|c|c|c|c|c|c|c|}
\hline \multirow[b]{2}{*}{ Distance between sections } & \multicolumn{8}{|c|}{ Metastasis size (diameter in $\mathrm{mm}$ ) } \\
\hline & 2.0 & 1.5 & 1.0 & 0.5 & 0.4 & 0.3 & 0.2 & 0.1 \\
\hline $2 \mathrm{~mm}$ & 0 (ITC) & 0 & 0 & 0 & 0 & 0 & 0 & 0 \\
\hline $1.5 \mathrm{~mm}$ & 1.32 & 0 (ITC) & 0 & 0 & 0 & 0 & 0 & 0 \\
\hline $1 \mathrm{~mm}$ & 1.73 & 1.12 & 0 (ITC) & 0 & 0 & 0 & 0 & 0 \\
\hline $0.5 \mathrm{~mm}$ & 1.94 & 1.41 & 0.87 & 0 (ITC) & 0 & 0 & 0 & 0 \\
\hline $0.25 \mathrm{~mm}$ & 1.98 & 1.48 & 0.97 & 0.43 & 0.31 & 0.17 & 0 & 0 \\
\hline $0.2 \mathrm{~mm}$ & 1.99 & 1.49 & 0.98 & 0.46 & 0.35 & 0.22 & 0 & 0 \\
\hline $0.15 \mathrm{~mm}$ & 1.99 & 1.49 & 0.99 & 0.48 & 0.37 & 0.26 & 0.13 & 0 \\
\hline $0.1 \mathrm{~mm}$ & 2.0 & 1.50 & 0.99 & 0.49 & 0.39 & 0.28 & 0.17 & 0 \\
\hline $0.05 \mathrm{~mm}$ & 2.0 & 1.50 & 1.0 & 0.50 & 0.40 & 0.30 & 0.19 & 0.09 \\
\hline
\end{tabular}

The cells that are below the stepped line correctly identify micrometastases as such. (For details, see table 1 .) ITC, isolated tumour cells. 


\section{Take home messages}

- Sentinel lymph nodes (SLNs) are the most likely sites of nodal metastasis

- Macrometastases have prognostic relevance and all should be identified, preferably correctly as macrometastases-levels taken $1 \mathrm{~mm}$ apart should be sufficient for this

- SLNs are also suited to studying the prognostic relevance of micrometastases-step sections taken 200 or $250 \mu \mathrm{m}$ apart are ideal for this purpose

metastasis. The detection of nodal involvement by means of IHC has been reported to be associated with the involvement of further echelon lymph nodes in around $10 \%$ of cases. ${ }^{6}$ Thus, the use of IHC should not be discouraged, but care should be taken when the results are interpreted, not only because of the well recognised potential pitfalls, but also because of the harm that can result from unduly upstaging breast cancers. ${ }^{19}$

In summary, on the basis of a simplified model of SLN metastasis, it is suggested that SLNs should be investigated at intervals of $1 \mathrm{~mm}$ to establish a reasonably reliable status without metastases measuring $>2 \mathrm{~mm}$, and the levels assessed should not be separated by more than 200-250 $\mu \mathrm{m}$ if micrometastases are to be identified. Obviously, this last sectioning protocol would be the one recommended in clinical practice, if both macrometastases and micrometasases were to be identified.

\section{ACKNOWLEDGEMENTS}

The author was supported by a János Bolyai Research Fellowship of the Hungarian Academy of Sciences and grant ETT 176/01 of the Hungarian Ministry of Health.

\section{REFERENCES}

1 Giuliano AE, Dale PS, Turner RR, et al. Improved axillary staging of breast cancer with sentinel lymphadenectomy. Ann Surg 1995;180:700-4.

2 Turner RR, Ollila DW, Krasne DL, et al. Histopathologic validation of the sentinel lymph node hypothesis for breast carcinoma. Ann Surg 1997;226:271-6

3 Czerniecki BJ, Scheff AM, Callans LS, et al. Immunohistochemistry with pancytokeratins improves the sensitivity of sentinel lymph node biopsy in patients with breast carcinoma. Cancer 1999;85:1098-103.
4 Sabel MS, Zhang P, Barnwell JM, et al. Accuracy of sentinel node biopsy in predicting nodal status in patients with breast carcinoma. J Surg Oncol $2001 ; 77: 243-6$.

5 Stitzenberg KB, Calvo BF, lacocca MV, et al. Cytokeratin immunohistochemical validation of the sentinel node hypothesis in patients with breast cancer. Am J Clin Pathol 2002;1 17:729-37.

6 Cserni G, Amendoeira I, Apostolikas N, et al. Pathological work-up of sentinel lymph nodes in breast cancer. Review of current data to be considered for the formulation of guidelines. Eur J Cancer 2003:39:1654-67.

7 Sobin LH, Wittekind Ch, eds. UICC TNM classification of malignant tumours, 6th ed. New York: John Wiley and Sons, 2002.

8 Cserni G. Complete sectioning of axillary sentinel nodes in patients with breast cancer. Analysis of two different step sectioning and immunohistochemistry protocols in 246 patients. J Clin Pathol 2002;55:926-31.

9 Dowlatshahi K, Fan M, Snider HC, et al. Lymph node micrometastases from breast carcinoma. Reviewing the dilemma. Cancer 1997;80:1 188-97.

10 Huvos A, Hutter RVP, Berg J. Significance of macrometastases and micrometastases in mammary cancer. Ann Surg 1971;173:44-6.

11 American Joint Committee on Cancer. Breast. In: Greene FL, Page DL, Fleming ID, eds. AJCC cancer staging handbook, 6th ed. New York: Springer Verlag, 2002:155-81.

12 Schwartz GF, Giuliano AE, Veronesi U, et al. Proceedings of the consensus conference on the role of sentinel lymph node biopsy in carcinoma of the breast April 19 to 22, 2001, Philadelphia, Pennsylvania. Hum Pathol 2002;33:579-89.

13 Millis RR, Springall R, Lee AHS, et al. Occult axillary lymph node metastases are of no prognostic significance in breast cancer. $\mathrm{Br} J$ Cancer 2002;86:396-401.

14 Torrenga H, Rahusen FD, Meijer S, et al. Sentinel node investigation in breast cancer: detailed analysis of the yield from step sectioning and immunohistochemistry. J Clin Pathol 2001;54:550-2.

15 Cserni G, Amendoeira I, Apostolikas N, et al. Discrepancies in current practice of pathological evaluation of sentinel lymph nodes in breast cancer. Results of a questionnaire-based survey by the European working group for breast screening pathology. J Clin Pathol [In press.].

16 Cserni G. Sentinel lymph node histopathology in breast cancer [letter]. Am J Surg Pathol 2002;26:1368.

17 Meyer JS. Sentinel lymph node biopsy: strategies for pathologic examination of the specimen. J Surg Oncol 1998;69:212-18.

18 van Diest PJ. Histopathological workup of sentinel lymph nodes: how much is enough? J Clin Pathol 1999:52:871-3.

19 Weaver DL. Sentinel lymph nodes and breast carcinoma. Am J Surg Pathol 2003;27:842-5.

20 Cserni G. Mapping metastases in sentinel lymph nodes of breast cancer. Am J Clin Pathol 2000;1 13:351-4.

21 Diaz LK, Hunt K, Ames F, et al. Histologic localization of sentinel lymph node metastases in breast cancer. Am J Surg Pathol 2003;27:385-9.

22 Cserni G. Histologic localization of sentinel lymph node metastases in breast cancer [letter]. Am J Surg Pathol 2004;28:275.

23 Cserni G. The potential value of intraoperative imprint cytology of axillary sentinel lymph nodes in breast cancer patients. Am Surg 2001;67:86-91.

24 Cserni G. The effect of increasing the surface sampled by imprint cytology on the intraoperative assessment of axillary sentinel lymph nodes in breast cancer patients. Am Surg 2003;69:419-23.

25 Teng S, Dupont E, McCann C, et al. Do cytokeratin-positive-only sentine lymph nodes warrant complete axillary lymph node dissection in patients with invasive breast cancer? Am Surg 2000;66:574-8.

26 Jakub JW, Diaz NM, Ebert MD, et al. Completion axillary lymph node dissection minimizes the likelihood of false negatives for patients with invasive breast carcinoma and cytokeratin positive only sentinel lymph nodes. Am J Surg 2002; 184:302-6. 